\title{
Nocturnal Polyuria with Abnormal Circadian Rhythm of Plasma Arginine Vasopressin in Post-stroke Patients
}

\author{
Ryuji SAKAKIBARA***, Tomoyuki UCHIYAMA*, Zhi LiU*, Tatsuya YAMAMOTO*, \\ Takashi ITO*, Tomonori YAMANISHI*** and Takamichi HATTORI
}

\begin{abstract}
Objective Nocturia is a common reason for interrupted sleep in post-stroke patients. These patients often have neurogenic bladder overactivity. However, little is known about the possible contribution of nocturnal polyuria in the patients.

Methods We measured the number of nocturia, the circadian plasma arginine vasopressin (AVP) level and urinary excretion in $\mathbf{4}$ patients with stroke.

Results All patients had nocturnal urinary frequency (three times in one and twice in 3). All patients were revealed to have nocturnal polyuria, and the ratio of nocturnal urinary output to 24 hour volume ranged from $36 \%$ to $63 \%$. Measurement of daily plasma AVP variation showed that all patients lost normal nocturnal rise of the plasma AVP concentration. Two patients were successfully treated with $5 \mu \mathrm{g}$ of intranasal desmopressin once a night, a potent analogue of AVP, without hypertension particularly in the night, signs of congestive cardiac failure or any electrolyte abnormality such as hyponatremia.

Conclusion Our post-stroke patients had nocturnal polyuria with abnormal circadian rhythm of plasma AVP secretion. Desmopressin reduced nocturnal waking in urination. It also ameliorated nocturnal dehydration that might trigger a stroke recurrence in the patients.

(Internal Medicine 44: 281-284, 2005)
\end{abstract}

Key words: stroke, nocturnal polyuria, circadian rhythm, arginine vasopressin, desmopressin

\section{Introduction}

A common reason for interrupted sleep in the general adult population is nocturia, which is waking during the night to urinate (1). Nocturia may present solely but also with other lower urinary tract (LUT) symptoms, such as urinary urgency or urge urinary incontinence. Among the factors that predispose one to the development of the LUT symptoms are neurogenic bladder overactivity and nocturnal polyuria. As compared to bladder overactivity that commonly results from lesions in the mediofrontal cortex and the basal ganglia $(2,3)$, nocturnal polyuria involves complexity of renal, cardiac and neural mechanisms $(4,5)$. However, study of brain in neurological patients with nocturnal polyuria indicated that there is a lesion of the suprachiasmatic nucleus (SCN) (6) that regulates arginine vasopressin (AVP)-containing neurons in the paraventricular nucleus (PVN) and the supraoptic nucleus (SON), which lead to impaired circadian rhythm of plasma AVP concentration that normally rises in the night (7). The underlying conditions for nocturnal polyuria include degenerative neurological disorders such as multiple system atrophy (7-9) and Alzheimer's disease (10), spinal cord injury $(11,12)$, and aging (13). However, little is known as to whether cerebrovascular diseases may cause nocturnal polyuria. Here, we describe our results of measurement of circadian plasma AVP level and urinary excretion in post-stroke patients. We also describe the effect of desmopressin (1-desamino-8-D-arginine vasopressin: DDAVP), a potent analogue of AVP (7), on nocturnal polyuria in a subset of the patients.

\section{Methods}

We recruited 4 post-stroke patients, all women, mean age 66 years, range $62-76$ years (Table 1). All patients were ambulant with or without an aid. The MRI and CT scans confirmed the location of lesions as multiple cerebral infarction in 2 with thalamo-hypothalamic lacunas, fronto-parietal infarction due to right middle cerebral artery occlusion in one, and left pontine infarction in one. None had apparent renal,

\footnotetext{
From *Department of Neurology, Chiba University, Chiba, **Department of Neurology, Kashima Rosai Hospital, Ibaragi and ***Department of Urology, Dokkyo Medical College, Tochigi

Received for publication July 14, 2004; Accepted for publication October 29, 2004

Reprint requests should be addressed to Dr. Ryuji Sakakibara, Neurology Department Chiba University, 1-8-1 Inohana, Chuo-ku, Chiba 260-8670
} 
Table 1. Patients and the Number of Nocturia

\begin{tabular}{ccclc}
\hline Patient & Age (years) & Sex & \multicolumn{1}{c}{ Diagnosis } & *Nocturia \\
\hline 1 & 62 & F & L pontine basis infarction & 2 \\
2 & 67 & F & multiple infarction & 2 \\
3 & 71 & F & R middle cerebral artery infarction & 2 \\
4 & 76 & F & multiple infarction & 3 \\
\hline
\end{tabular}

*Nocturia: times per night.

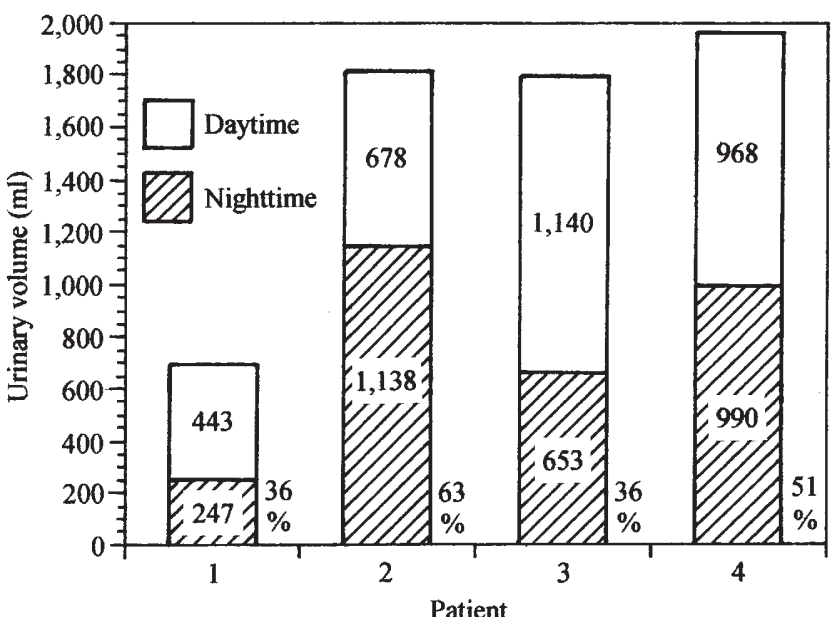

Figure 1. Daily urinary output in post-stroke patients. Urinary volume in the daytime (6 AM to $10 \mathrm{PM}$ ) and the nighttime (10 PM to 6 AM) was measured for 5 succeeding days and the mean value of each parameter is shown. All patients had nocturnal polyuria, in which the urinary output in the nighttime was more than $33 \%$ of the total 24 hour volume.

hepatic or cardiac diseases that might affect urinary secretion, and none was taking diuretic drugs. All patients had normal sleep-waking cycles. The study was made at least 4 weeks after an episode of stroke in each patient. First, urinary volume in the daytime (6 AM to $10 \mathrm{PM}$ ) and the nighttime (10 PM to $6 \mathrm{AM}$ ) was measured for 5 successive days, during which the number of nocturnal urination was counted. After these measurements, the average of each parameter was calculated. Nocturnal urinary frequency (nocturia) is defined as the number of urination during sleep in the nighttime as twice or more. Nocturnal polyuria is defined as the urinary output in the nighttime is more than $33 \%$ of total 24 hour volume (4). Secondly, the plasma AVP concentration was measured every 4 hours from $8 \mathrm{AM}$ in the morning to 8 AM in the next morning, each measuring after 15 minutes' bed-rest, looking for a circadian rhythm of the plasma AVP level which normally rises in the nighttime, i.e., almost twofold as the baseline (14). All patients gave informed consent before participating in the study.

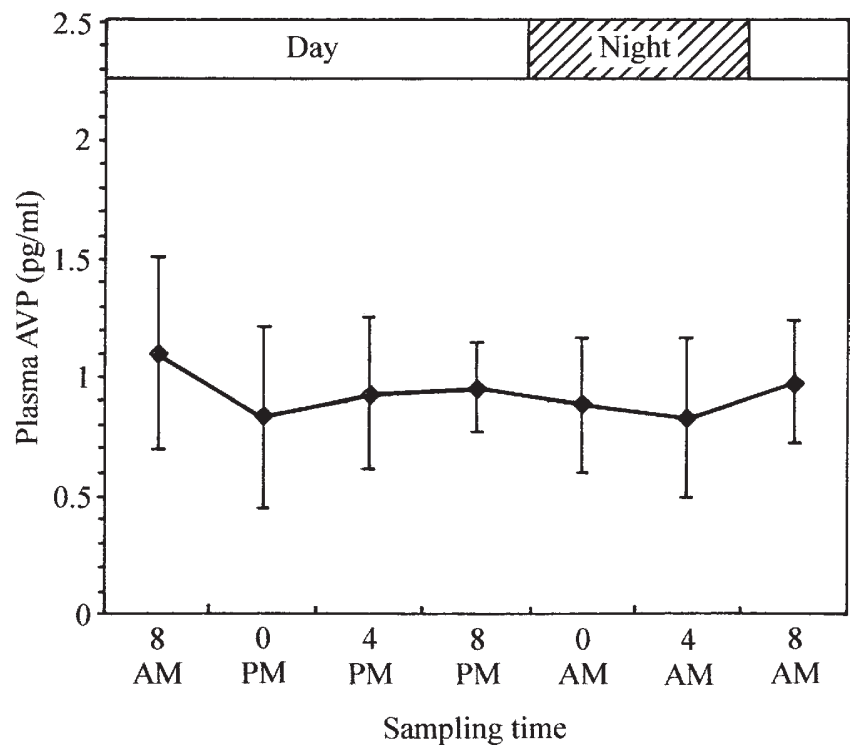

Figure 2. Diurnal variation of the plasma AVP concentration in post-stroke patients. These patients lost the normal nocturnal rise of the plasma arginine vasopressin (AVP) concentration. Mean \pm standard deviation was plotted.

\section{Results}

All patients had nocturnal urinary frequency (three times in one and twice in 3), which seemed to appear after the occurrence of stroke. In addition, all patients had nocturnal polyuria, and the ratio of nocturnal urinary output to 24 hour volume ranged from $36 \%$ to $63 \%$ (Fig. 1). Measurement of the daily plasma AVP variation showed that all patients lost circadian rhythm of the plasma AVP concentration (Fig. 2), which normally increased in the night. After the assessment, two of the patients (cases 3,4) and their spouses requested to use $5 \mu \mathrm{g}$ of intranasal desmopressin once a night. We taught them how to use it and possible adverse events in detail, and observed them for 8 weeks during hospitalization and outpatient clinic, with regards to serum chemistry, electrolytes and cardiovascular function. During an observation period, the nocturnal polyuria successfully lessened in the patients (case 3: $36 \%$ to $25 \%$, case $4: 51 \%$ to $38 \%$ ). Both patients came to sleep better because of a reduction in the nocturia (case 3 : 
twice to once, case 4: 3 times to once or twice) when on desmopressin. None of them showed hypertension particularly in the night, signs of congestive cardiac failure or any electrolyte abnormality such as hyponatremia.

\section{Discussion}

There is a circadian rhythm of urinary flow in normal children over 7 years and in adulthood (4). The ratio of daytime to nighttime urine production is usually $>2: 1$, so that $33 \%$ or less of daily urine output occurs during sleep. AVP, or antidiuretic hormone, is the major hormone responsible for the regulation of urine formation. In healthy adults, diurnal release of AVP into plasma has its peak during nighttime (14). Loss of the AVP circadian rhythm occurs in congestive heart failure, nephrosis, and cirrhosis with ascites (14). Central nervous system lesions affecting the hypothalamicposterior pituitary system also cause nocturnal polyuria [loss of AVP circadian rhythm due to the regulatory centre (SCN) lesion](6) and diabetes inspidus [loss of AVP secretion due to the secreting neuron (SON/PVN) lesion] (15). The present study revealed that all of our post-stroke patients lost circadian rhythm in the urinary excretion and in the AVP secretion into plasma. None of the patients had apparent renal, hepatic or cardiac diseases that might affect urinary output. The MRI and CT scans of the patients showed multiple cerebral infarction with thalamo-hypothalamic lacunas in 2 . Other patients had no apparent lesion in the hypothalamus. However, the hypothalamic neurons have fibre connection with the frontal cortex and receive catecholaminergic projections from the pons and the medulla (16), which are lesioned in the patients. These ischemic lesions, and neuronal degeneration by ageing as well (13), may contribute to the loss of circadian rhythm in the AVP secretion in our post-stroke patients. However, in our patients, the impaired circadian rhythm was not so widespread since the sleep-waking cycle was maintained. Although the number of our patients was small, nocturnal polyuria most frequently resulted in nocturnal urinary frequency. It interrupts sleep of patients and their spouses, and may lead to accidental falls. Nocturnal polyuria also causes nocturnal dehydration, which may trigger a stroke recurrence in the patients. Desmopressin is a potent analogue of AVP (hypertensive and antidiuretic effects: 100, 100 in AVP; 0.39, 1,200 in desmopressin, respectively) (17) and widely used in the treatment of nocturnal enuresis in childhood (4) and nocturia of various aetiology $(18,19)$. However, 20-40 $\mu \mathrm{g}$ use of desmopressin may have adverse effects resulting from water retention and hyponatremia, particularly in the elderly patients $(20,21)$. Therefore, we prescribed $5 \mu \mathrm{g}$ of intranasal desmopressin once a night in 2 patients with nocturnal polyuria. Although a small amount of each dose, desmopressin treatment improved the nocturnal polyuria in both patients together with an improvement of nocturnal urinary frequency. Both patients did not show hypertension, hyponatremia or other adverse effect during an observation period.
In conclusion, post-stroke patients may have a loss of circadian rhythm in AVP secretion and urinary excretion. Desmopressin successfully reduces nocturnal waking in urination. It also ameliorates nocturnal dehydration that might trigger a stroke recurrence in the patients.

\section{References}

1) Hetta I. The impact of sleep deprivation caused by nocturia. BJU Int 84 (Suppl 1): 27-28, 1999.

2) de Groat WC, Booth AM, Yoshimura N. Neurophysiology of micturition and its modification in animal models of human disease. in: Nervous Control of The Urogenital System. Maggi CA, Ed, The Autonomic Nervous System, vol 3, Chur, London, Harwood Academic Publisher, 1993: 227-290.

3) Sakakibara R, Fowler CJ. Cerebral control of bladder, bowel, and sexual function and effects of brain disease. in: Neurology of Bladder, Bowel, And Sexual Function. Fowler CJ Ed, Butterworth-Heinemann, Boston, 1999: 229-243.

4) Norgaard JP, Djurhuus JC, Watanabe H, Stenberg A, Lettgen B. Experience and current status of research into the pathophysiology of nocturnal enuresis. Br J Urol 79: 825-835, 1997.

5) Weiss JP, Blaivas JG. Nocturia. J Urol 163: 5-12, 2000.

6) Ozawa T, Oyanagi K, Tanaka H, et al. Suprachiasmal nucleus in a patient with multiple system atrophy with abnormal circadian rhythm of arginine vasopressin secretion into plasma. J Neurol Sci 154: 116-121, 1998.

7) Mathias CJ, Fosbraey P, daCosta DF, et al. The effect of desmopressin on noctumal polyuria, overnight weight loss, and morning postural hypotension in patients with autonomic failure. Br Med J 293: $353-$ 354, 1986.

8) Ozawa $T$, Tanaka H, Nakano $R$, et al. Nocturnal decrease in vasopressin secretion into plasma in patients with multiple system atrophy. J Neurol Neurosurg Psychiatry 67: 542-545, 1999.

9) Sakakibara R, Matsuda S, Uchiyama $T$, et al. The effect of intranasal desmopressin on nocturnal waking in urination in multiple system atrophy patients with nocturnal polyuria. Clin Auton Res 13: 106-108, 2003.

10) Ouslander JG, Nasr SZ, Miller M. Arginine vasopressin levels in nursing home residents with nighttime urinary incontinence. J Am Geriatr Soc 46: 1274-1279, 1998.

11) Szollar S, North J, Chung J. Antiuretic hormone levels and polyuria in spinal cord injury; a preliminary report. Paraplegia 33: 94-97, 1995.

12) Kilinc $S$, Akman $M N$, Levendoglu $F$, et al. Diurnal variation of antidiuretic hormone and urinary output in spinal cord injury. Spinal Cord 37: 332-335, 1999.

13) Nakamura S, Kobayashi Y, Tozuka K, et al. Circadian changes in urine volume and frequency in elderly men. J Urol 156: 1275-1279, 1996.

14) George CPL, Messerli FH, Genest J, et al. Diurnal variation of plasma vasopressin in man. J Clin Endocrinol Metab 41: 332-338, 1975.

15) Hoshimaru M, Hashimoto N, Kikuchi H. Central diabetes inspidus resulting from a nonneoplastic tiny mass lesion localized in the neurohypophyseal system. Surg Neurol 38: 1-6, 1992.

16) Lightman SL, Williams TDM. Hypothalamic function in autonomic failure. in: Autonomic failure; a textbook of clinical disorders of the autonomic nervous system. Bannister R Ed, Oxford University Press, Oxford, 1988: 381-392.

17) Shulman LH, Miller JL, Rose LI, et al. Desmopressin for diabetes insipidus, hemostatic disorders and enuresis. Am Fam Physician 42: 1051-1057, 1990

18) Hoverd PA, Fowler CJ. Desmopressin in the treatment of daytime urinary frequency in patients with multiple sclerosis. J Neurol Neurosurg Psychiatry 65: 778-780, 1998.

19) Asplund R, Sundberg B, Bengtsson P. Oral desmopressin for nocturnal polyuria in elderly subjects; a double-blind, placebo-controlled ran- 
SAKAKIBARA et al

domized exploratory study. BJU International 83: 591-595, 1999.

20) Ferrer J, Halperin I, Conget JI, et al. Acute water intoxication after intranasal desmopressin in a patient with primary polydispsia. J
Endocrinol Invest 13: 663-666, 1990.

21) Cannon A, Carter PG, McConnel AA, et al. Desmopressin in the treatment of nocturnal polyuria in the male. BJ U Int 84: 20-24, 1999. 